\title{
Village Voices: Lessons about Processes for Disease Prevention from a Qualitative Study of Family Health Leaders in a Community in Northeastern Thailand
}

\author{
D Jongudomkarn ${ }^{1 *}$, P Singhawara ${ }^{2}$, C Macduff ${ }^{3}$
}

\begin{abstract}
Background: Cancer is a primary source of concern in Thailand and other countries around the world, including the Asian-Pacific region. Evidence supports that an important contributing cause of cancer and other chronic illnesses such as stroke, diabetes, and hypertension is excessive alcohol consumption. Studies conducted in Thailand reveal a worrisome rise in the number of new and regular drinkers in communities. Therefore, actions for primary, secondary and tertiary prevention of problem drinking are necessary. In recent years nurses in North East Thailand have been developing and implementing the Khon Kaen Family Health Nursing model to embed disease prevention in communities through the actions of family health nurses and local family health leaders. Aim: The aim of this qualitative research was to better understand the experiences of the local family health leaders using this model and to synthesize lessons learned. Materials and Methods: As part of a participatory action research approach involving analysis of focus group discussions and individual interviews, the experiences of 45 family health leaders were synthesized. Results: Four main themes were identified, namely: $i$ ) Family first: role modeling beginning at the personal and family level. ii) Local leverage: using village community forums to reduce alcohol drinking. iii) Gentle growth: making the first step and treading gently; and $i v$ ) Respect, Redemption, Rehabilitation: valuing the person to re-integrate them in the village society. Conclusions: As alcohol consumption in the village declined significantly following the prevention program, these findings illuminate how low-tech integrated prevention approaches may be very useful, particularly in rural communities. The lessons learned may have relevance not only in Thailand but in other countries seeking to prevent and mitigate behavior that conduces to diseases such as cancer.
\end{abstract}

Keywords: Family health leaders - lessons learned - the Khon Kaen university family health nursing model

Asian Pac J Cancer Prev, 16 (10), 4401-4408

\section{Introduction}

Cancer is a leading cause of illness and death throughout the world (WHO, 2010; CAS Board, 2014; Gupta and Johnson, 2014; Oh et al., 2014; Arora et al., 2015). Excessive regular consumption of alcoholic beverages is a known risk factor which can lead to mouth cancer, laryngeal cancer, esophageal cancer, liver cancer, colo-rectal cancer and breast cancer, according to studies conducted by the World Health Organization (WHO, 2010) and recent meta-analyses (de Menezes et al., 2013). Indeed The World Health Organization has estimated that the intake of excess alcohol is involved in approximately a quarter of all health destroying activities or problems (WHO, 2005).

Cancer is a significant health problem and the leading cause of death in Thailand (Wirasorn et al., 2012; Chindaprasirt et al., 2014; Jongudomkarn, 2014; Leenharattanarak and Lertkhachonsuk, 2014).
Concurrently concerns are growing about the increasing use of alcohol and the consequences it is likely to have in terms of causing such cancers and also a range of chronic diseases (CAS Board, 2014). This paper reports findings from a qualitative study of the experiences of family health leaders who were involved in the development and implementation of a family and community social capital based approach which aimed to reduce the use of alcohol. As any such approach requires to be culturally appropriate and sensitive, it is firstly useful to consider Thai culture and its relationship with alcohol.

Context: In Thailand, alcoholic beverages have been related with the traditions and customs of the people since historic times. Drinking alcohol can be a part of life style and social activities that include traditions, trading and political and health aspects. A study concerning the alcohol drinking behavior of Thai people, conducted in Thailand during the year 2010 (CAS Board, 2010), found that 7 million Thai people aged 15 years and older $(32 \%$ of that

${ }^{1}$ Center for Research and Training on Gender and Women Health (CRTGWH), Faculty of Nursing, Khon Kaen University, ${ }^{2}$ Nurse Manager, Northeastern-Watana Hospital, Thailand, ${ }^{3}$ School of Nursing and Midwifery, Robert Gordon University, Aberdeen, Scotland *For correspondence:darjon@kku.ac.th 
age group) drank alcohol. This was from the country's total population of 53.9 million people. Five times the number of men drink alcohol than the number of women who drink alcohol; men started to drink at an average age of 19.4 years old; and women started at an average age of 24.6 years old. Significantly, over the past 11 years the number of people within the age group of 15-19 years old who drink alcohol regularly has risen by $70 \%$. At this rate of increase, within forty years, 2 out of every 3 youths will regularly be drinking alcohol.

Indeed a report by the World Health Organization (2010) has shown that Thailand is one of the top ten consumers of alcohol in the world and is the third largest alcohol consuming country within the Asian region, after Japan and South Korea. The alcohol consumption rates in the different regions in Thailand are as follows: $22.6 \%$ in the northern region; $22.2 \%$ in the northeastern region; $23.7 \%$ in the middle region; $21.1 \%$ in the southern region; and $10.4 \%$ in Bangkok. The statistics show the northeastern region of Thailand had the third highest rate of alcohol consumption. However, the rating is close to the same as that of the middle and the northern regions which are also mainly agricultural societies. It is thought that the life style and social aspects of agricultural communities encourages people to drink because they believe that whisky is presented to workers in appreciation for their help. Whisky also is used in ceremonies to vow to the spirits or other supernatural beings asking for protection, to help conserve and protect their valuables, and to wish for the best yields in agriculture production. The whisky in used as a relaxant after being tired from working, as a greeting and welcome to guests or relatives who come to visit, and is used in a variety of celebrations.

While moderate usage in these social and cultural contexts may be seen to have some positive aspects, regular excessive alcohol consumption negatively affects the people who drink, their family members, and wider society as well. In Thailand, the Alcohol Use Disorders Identification Test (AUDIT), developed by WHO, was conducted for detecting alcohol use disorders or risky drinking in targeted population. Risky drinking was defined as heavy drinking (280 g pure alcohol/week for men and $168 \mathrm{~g}$ pure alcohol/week for women) or binge drinking (consumption of $60 \mathrm{~g}$ pure alcohol on $\geq 1$ occasion per week) (Silapakij and Kittirattanapaibul, 2009).

Within this context many organizations in Thailand have started to campaign to highlight the harmful and negative effects from alcohol. This has involved various societal primary prevention strategies including launching rules to control alcohol sales in order to help reduce alcohol consumption. Moreover, health professionals such as nurses have increasingly become engaged in primary, secondary and tertiary prevention work to address this problem.

\section{The family health nursing model}

One approach which seeks to combine and integrate these three types of work is the Khon Kaen Family Health Nursing Model (KKU FHN model, Figure 1). This model is informed by the World Health Organization Europe family health nurse model (WHO, 2000) which incorporates health care within four dimensions; $i$ ) Health promotion or disease prevention; ii) Early risk detection; iii) Direct/Care, thereby solving or reducing sickness and crisis; and $i v$ ) Rehabilitation. As Figure 1 shows, these dimensions are at the heart of the model, and are framed within an ethos of participatory action where nurses work with, and within, local communities to help foster and harness the benefits of social capital. In turn families are seen as the "engine room" for this community capital.

Work is conducted by following the family nursing process and then making a connection for providing care throughout the community, thereby extending participation and encouraging families in the community to look after themselves and each other. The process involves the family health nurses and other health care providers using eight action strategies. These action strategies consist of: $i$ ) Finding the responsible host and health leaders and eliciting relevant needs and issues for targeted work operating in the community; ii) Proposing the issues and then conducting training for family health leaders concerning the health aspects for which they want to campaign, so they can access the targeted population according to their level of understanding and their perception of how important the issue is; iii) Targeting caring health leaders, which has proven to be an important driving force to be able to conduct campaigns in targeted areas; iv) Disseminating reliable information to all family health leaders so they are consistent in following the campaign guidelines in their areas of responsibility, and sharing the responsibility to cover all in-depth situations occurring in their areas; $v$ ) Responsibility starts as a function of the family health leaders and spreads to the health stations of the areas involved. This depth of participation helps to prevent or mitigate any risk situation that might occur; vi) The family health leaders function in risk reduction within their areas of responsibility, and also coordinate their efforts with the nurses for reducing risk situations before problems begin or spread; vii) Rehabilitation is conducted by the family health leaders within their areas of responsibility, and includes rehabilitation and in-depth care coordinated with health care providers / nurses; and viii) Sustaining progress is made possible by the health insurance associations and budget support supplied by each area's health clinics. The different areas coordinate their operations by drafting a strategic plan, performance plan and assessments. Health care providers/nurses in the area stations, under the primary care unit (PCU) administration, act as the facilitators or assistants for the performances and the outcome expectations which were family wellbeing, family risk reduction, patients being referred to the appropriate caring system and getting rehabilitation.

This model has been developed and modified, for example through research into the perceptions of family health nurses themselves (Jongudomkarn and Macduff, 2014). The model's usefulness in mobilization to address alcohol issues has recently been highlighted by Jongudomkarn and others (2013a; 2013b), focusing on the experiences of 10 female volunteer participants in the 8 areas in 7 provinces of North East Thailand. In this article 
we now report a related qualitative study in a specific area (Loei province) which sought to better understand the experiences of 45 local family health leaders who worked with family health nurses guided by this evolving model. This is important as the particular project that these leaders were involved in appears to have had successful outcomes in terms of its key goal which was to reduce the consumption of article in a particular village. Thus the study aims to illuminate the process of how these family health leaders contributed to this success.

Project and setting: This research was one of a group of research projects and studies being conducted in several areas during the same period. Intervention to reduce consumption of alcohol and thereby help towards the prevention of cancer, long term health conditions and social problems was proposed as the key goal of the project. Before starting these projects a survey of alcohol consumption and health problems in the area, including the participating communities, was conducted.

All participants in the current study lived in the same village community within one of the rural areas in the upper part of northeastern Thailand (Loie province). The population of the village community totals 926 people: 409 males and 517 females, living in 244 households. The occupation of most people in the community is farming, and a variety of plants are cultivated including sugar cane, corn, cassava and rice. Another source of supplemental income is lottery sales. Vendors leave the community two times every month, and return to the villages for ten days to rest and wait for the next time to leave before the start of the next lottery. This way of working necessitates the parents to leave the children with the grandfather or grandmother to look after them. This often causes problems because the grandparents are not always capable of providing all care needed, the children often act up, and the grandparents allow them to do things they shouldn't do. Alcohol is an integral part of a number of cultural events and local traditions, but unfortunately over-consumption can be a problem at such events and this is often associated with violence. Over the years, many of the working age villagers have emigrated to work in other provinces and the permanent village population is predominantly the elderly, females and children.

\section{Materials and Methods}

The initial survey findings concerning people's alcohol consumption and health risks were presented to all community leaders. Family health leaders who participated in the project were nominated and recruited via community forums and recommendations from the district administration organization (Aor. Bor. Tor) and community leaders. The total number of people who participated was 45 (31 females and 14 males), with an average age of 32-70 years old. All spoke the Isaan dialect and all were married. Their occupations included 5 government officers, 10 farmers, 20 vendors, and 10 laborers. The participatory action research project, approved by the Khon Kaen University Ethics Committee for Human Research, was conducted from February 2012 to February 2013.
The intervention part of this project commenced with the community forum process (stages 1 and 2) described above and followed the remaining six stages, maintaining an embedded participatory action research approach throughout. This meant that the villagers were active participants in defining, understanding and addressing the perceived issues and this concurrently involved active participation in generating the research data, as facilitated by a key link person from the project team.

As appropriate to generating understanding of the family leader's experience, this data was qualitative in nature comprising: observation field notes; eight focus group discussions (two initial groups with males; three initial groups with females; and return interviews with one male group and two female groups to member check the emergent themes); and 49 individual interviews (one with each of the 45 family leaders and further interviews with four of these leaders (three female and one male) who had emerged as most influential).

The key link person from the team was the second author who conducted the in-depth interviews and focus group discussions throughout the running period of the project. Prior to the field data collection, the first author trained her to pilot the observation and interview procedures. The research assistant undertook the participant observation and interviews during a continuous period of field and home visits (Yin, 2009). Detailed field notes were written down, reflected upon, discussed, and then interpreted with the first author as a process of ongoing reflection and sense making (LeCompte, 2000). The five main focus groups sought to identify broad topics and issues that were common to the family leaders' experiences. The main 45 semi-structured interviews with individuals firstly gathered demographic data, then explored experiences of the leaders with a view to achieving answers to the following two main questions: "What are the lessons learned about effective strategy that the team develops and adopts to promote family and community members' health and well-being?" and "What have you learned overall through your volunteer work?" The individual interviews allowed participants the chance to voice aspects that they may have been more reluctant to talk about in a group. Finally, the three focus groups for member checking presented the emergent themes of the research and invited feedback (Morgan, 1998; Silverman, 2000).

At the end of project intervention, quantitative data were obtained in relation to alcohol consumption. A reduction in the number of alcohol drinkers was noted, and data analyses also revealed decreased alcohol consumption in the community as a result of the health volunteer participants' interventions. The pre- and postintervention scores of the 76 participating villagers (58 males and 18 females) on the World Health Organization's (WHO) Alcohol Use Disorder Identification Test (AUDIT) (Silapakij and Kittirattanapaibul, 2009) showed a statistically significant decrease in post-intervention $(\overline{\mathrm{x}} \pm \mathrm{SD}=9.684 \pm 6.632)$ AUDIT scores compared with preintervention $(\overline{\mathrm{x}} \pm \mathrm{S} . \mathrm{D}=6.039 \pm 4.482)$ scores (paired t-test, $\mathrm{p}<0.001)$.

These quantitative findings are reported in detail 
elsewhere (Jongudomkarn et al., 2013a; 2013b) and are not the focus of the present paper. However they are important to note as they provide the context for understanding, from the family health leaders' point of view, how and why the project worked.

\section{Data analysis}

Data analysis of these audio recorded interviews and focus group discussions was conducted in four separate groups, undertaken with assistance from three research assistants who were Master's degree students in family nursing. Detailed notes of important issues, with typical examples, were written down from the audio recordings for use in subsequent sessions (Lincoln and Guba, 1985). Qualitative content analysis (Bryman, 2001) was employed by the first author and research assistants and this led to synthesis of the four main emergent themes.

\section{Rigor and trustworthiness}

The criteria of credibility, transferability, dependability and confirmability, as posited by Lincoln and Guba (198v) underpinned the approach to data collection and interpretation. In terms of credibility, there was prolonged and persistent engagement by the project team and particularly the research assistant in the field. As indicated above, this included peer debriefing and member checking. The triangulation of methods for completeness resulted in "thick description" (Lincoln and Guba, 1985), enhancing confirmability and potential transferability. The data generation and analysis produced an audit trail that enhanced dependability.

\section{Results}

The family heath leader participants spoke with pride of their willingness to help families and the community to reduce problems of alcohol consumption and to continue operating the program in the future. As one of the participants said: "We learn with lessons, working every day, and getting practice. The lessons learned could be very useful to improve our best practices."

Four main themes emerged from analysis of these leaders' experiences.

i) Family first: role modelling beginning at the personal and family level.

The project operation started by obtaining participating family health leaders' consensus as to how best and efficiently to distribute information and promote reduction and abstinence from alcohol consumption. Since villagers had seen alcohol consumption as a long time and regular behavior integrated into their life styles, change in thinking and behavior was seen as having to be gradual, starting with the individual, family and close friends and neighbors. This involved acting as role models for discussing and advising other people. Included in this strategy was the use of the family as the focal point, reflecting family as the essential building block of society. The family unit was seen to act as provider of lessons learned, transfer knowledge, and translate cultures and trends to the family members. In the words of several participants:

"We must manage our own family before other families. If our family can't change then it is difficult to tell other people and they will not believe us." (Miss Chorchang, age 44 years old)

"Do well inside our home before starting to promote the ideas to other people. If we cannot do well, how can we teach other people? Every committee member must look after their own family's health situation." (Mr. Kor, age 50 years old).

"Concerning myself: my son had given up, and I had given up for ten years. My youngest son had also given up, and so did some of our neighbors." (Mr. Khorkhowy,
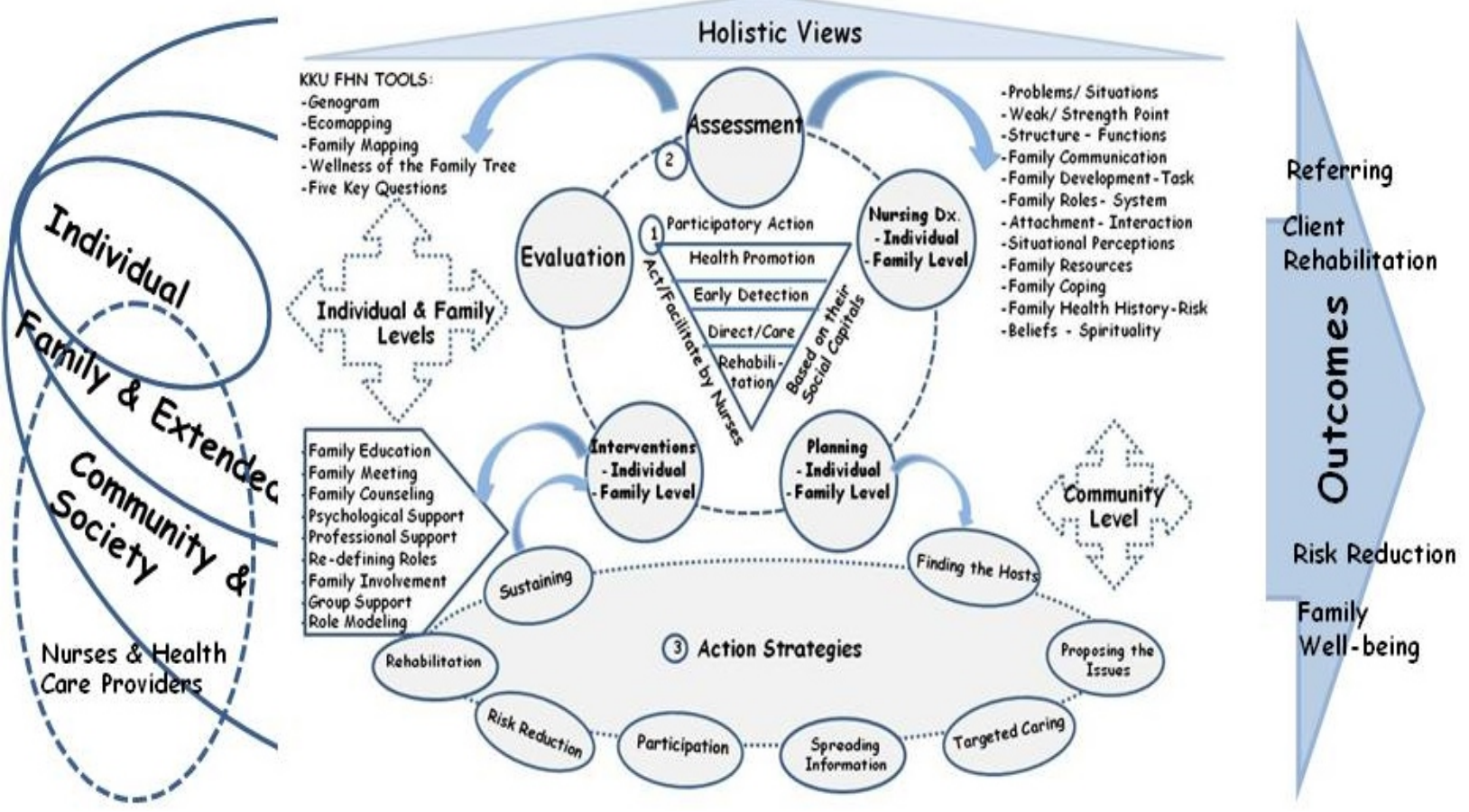

Figure 1. The KKU Family Health Nursing Model 
age 70 years old)

"Talking to one person does not produce any results. For example, if I have a meeting with a family I talk to the husband as the head of family and also include the wife and children. Each family member has a vote and whichever condition or items have the most votes, the other members must follow. If the wife and children agree then the husband cannot disagree." (Mr. Jorjarn, age 49 years old)

ii) Local leverage: using village community forums to reduce alcohol drinking.

A community forum was held at the studied site every month to exchange information with the people in the community. This opened up the opportunity for people to meet and talk, to develop and establish guidelines, and to work on other problems together. As an example: the situation of violence among people in the community was increasing and was causing damage to personal and public property. Because of this the community agreed, via the forum, to initiate new rules for damage claims. People in the community agreed that whoever causes damage must either repair the damage themselves or must pay the cost of the needed repairs to the property owner. In the case of personal violence or fighting among village members, each must pay 250 baht then go to the home of the village leader for resolution of the conflict. Money collected from the event would be saved in the village account. The rules which were agreed upon and started by the community forum would apply strictly and fairly to everybody in the community. In the words of the participants:

"If the first and the second households have done this, then the third and the forth must follow them as well" (Miss Ngorngoo, age 61 years old).

"I was one person comprising the community. If the others agree then I will follow because it doesn't affect only me but also many other people. Whatever they do I will follow too." (Mr. Phorphun, age 64 years old)

"If most other people have given up, we will turn around to look at those others, feel ashamed, and finally give up ourselves." (Mr. Choorching, age 56 years old)

"For example if people surrounding have given up alcohol drinking by the campaign, and only one family remains drinking, maybe they will feel ashamed to continue drinking alcohol. Use most people as the modelers to convince the small remaining portion of people. They will start to think about how other villages have done well, so why does our village really like to drink? Some people get by living on 40,000-50,000 bath, while others spend100,000 -200,000 baht because of their alcohol drinking; I think they will start to think about that. However, I think never mind if other people do not consider this yet because we are piloting the program. If this person does not accept the program yet but still other people are accepting it; if people surrounding them stop drinking but that house is still doing it, I think just let them drink." (Mr. Ngorngoo, age 60 years old)

"I do talk to them to participate in the project, but the things I mention could affect them. They said "Oh, I want this area to have no alcohol", but I do not say to them to stop drinking. Yet somehow they will be affected by the program. If they are continuing to drink they will feel ashamed eventually." (Mr. Khorkhai, age 60 years old)

"In the future people will accept it. Once it does start it will become a community tradition." (Miss. Khorkhai, age 41 years old)

As can be seen from the comments above, local leverage was exerted through peer pressure and shame was a prominent dynamic within this context.

iii) Gentle growth: making the first step and treading gently

Some participants referred to a Thai proverb that says "A great person is the one who does a thing for the first time". Others mentioned to Lao Tzu's teaching. Lao Tzu was the famous Chinese wise man who said "The journey of a thousand miles begins with one step". The rural society life styles in the communities maintain the character and quality of life of people by helping each other. Their life style comes from people's beliefs and popular traditions. These attitudes and beliefs are deeprooted in people's lives and translated from one generation to the next generation. They may or may not be aware of this process, but they are already following the pattern of conduct. For these reasons the participants who are leaders to drive the project explained that they needed to present their lessons step-by-step so that the villagers gained the opportunity to slowly adapt the new ideas into their way of life.

"Some people have the opinion that it can be done very rapidly, but time is needed to adjust." (Miss. Choorching, age 57 years old)

"Alcohol is involved in most of the funerals. I think it is sad during the funeral, so why do they have alcohol in order to enjoy the event? Some people do not have alcohol during a funeral. I don't ask why. Yet others have alcohol only during the Krathin festival. Some households choose to have alcohol involved with only one event, depending on their own pleasure, and we cannot change them." (Mr. Khorkhai, age 57 years old).

"We cannot tell them to give up right away. But we should convince them to reduce drinking from a full big bottle of whisky reduced to half of a bottle, then to a small bottle and then half of a small bottle, in that order. If we tell them to stop right away, I think it will cause a big conflict. One person said you shouldn't drink, but another one is sneaking to the shop to buy for their drinking." (Mr. Jhorjarn, age 49 years old).

"Right now, concerning my despondent feelings, I don't have any. We should continue doing this slowly because if we speed up people might give up. If we go too fast and strain, little by little we will sometimes loose and sometimes get tied up. Sometimes we need relief, like the ropes as well. If the ropes are hanging very loosely we will pull up but cannot strain it, since if it over strains it will tear apart with the sound of Phin (music instrument). If we continue to adjust the sound of music then we will regain all the melody of the music." (Mr. Korkai, age 50 years)

iv) Respect, Redemption, Rehabilitation: engaging with the person to re-integrate them in the village society

Through the project, the family leaders came to understand how valuing those who deviate from social norms could be very helpful. People who were excessive drinkers were not criminals but were often victims, 
needing help from society. Engaging with people, giving them respect, and complimenting them for their successes, emerged as valuable. Nevertheless, the motivations provided by fear or shame were also part of this mix. For example, some comments from participants are:

"We should gather to sit and talk together along with those who are drinking whiskey, not allowing them to go anywhere and talking formal with them. Try to stay beside them and let them talk with people who were not drinking whisky, and probably they will forget to drink finally." (Mr. Khorkhai, age 57 years old)

"If somebody can pull them into a good way, they will quit or not drink as frequently because nobody is drinking with them." (Mr. Jorjarn, age 49 years old)

"I knew someone who was always drinking whisky and getting smashed drunk, but later that guy gave up drinking whisky because he joined the committee of the village then got elected to be district administrator. The first year after being elected that guy was still drinking and then later rarely drank, and in the third year that guy finally stopped drinking." ( Mr. KhorKhai, age 57 years old)

"I am still drinking, but now I am slowly reducing drinking. Before I used to get smashed drunk, as Mr. Vee had mentioned. Since I became a leader and as my level increases, I cannot go back to doing the same thing. Maybe within one or two years I will probably stop, as Mr. Vee said.” (Mr. Jorjarn, age 49 years old)

\section{Discussion}

This paper reports the findings of a qualitative study of the experiences of 45 family health leaders, conducted in one community in northeastern Thailand. This participatory action research study was undertaken with respect for the tacit knowledge and experiences of the villagers (Hetherington, 2011) in their community, and sought to synthesize data from them with a view toward learning and greater understanding both locally and in a global perspective.

The key themes identified resonate with knowledge emerging within this part of Asia. The primacy of family role modeling and support is consistent with research results by McMorris and others (2011) who found that parents' supervised alcohol use or early-age alcohol use will reduce the development of adolescent alcohol problems.

However, the current study adds a distinct dimension in its highlighting of local community leverage. The sounding out of agreements by voices of the majority of the people through the community forums highlighted normative values. In turn this tended to put some pressure on those not conforming and related feelings of shame were frequently mentioned. It is important to note that recognition and use of strategies that invoke negative emotions like guilt are not usually explicitly supported in contemporary health promotion in western countries, yet nevertheless may be part of the realpolitik of work on the ground (e.g. working with mothers and fathers who smoke in their child's bedroom). In this regard it is also important to note that Thai society is rather more compliant and conformist in many aspects of life and that the negative aspect is in this case tempered with information giving and an approach that values the person.

This step by step, growth approach allowed people to have the opportunity to adjust their inner feelings and attitudes for more sustainable results (Lewis and Hurd, 2011; Jongudomkarn, 2014). In our study we found that stories told by individuals, by family members, and practical exercises and reviews allowed change to appear steadily, and in this regard the Thai virtue of patience was helpful.

As highlighted above, a key element in this was showing people respect and offering prospects for reintegration to the village society. This resonates with study results by Lizárraga and others (2007), which found that the factors which affect peoples' decision to participate in the community consist of: factors which demonstrate to people that there will be a return from what they have done, and factors that show opportunities that may be available to them through participation. The benefits they see as returned or received after participation are: regaining a place of standing in the society; new opportunities in an alcohol-free environment; the opportunity to prove personal value; and the ability to give and receive love. These factors agree with results reported in a study by Schäfer (2011), which found that the majority of participants had experienced painful and traumatic childhoods in their families of origin, which contributed to their subsequent addictive behaviour and which they felt had affected their current familial relationships. Thus the most important points for mending addictive problems in the community are: looking after each other in the community; establishing rules of social norms; forgiveness; giving opportunities; giving love and attention; and providing work which is suitable.

Hence, the qualitative findings elicited from these 45 family health leaders both confirm and expand relevant knowledge of how we might best work with, and in, Thai communities to tackle problems such as alcohol consumption and thereby reduce risk of cancer. These findings from community leaders add to those reported recently form a related initiative in a different region (Jongudomkarn, 2014).

From the point of view of the research and development team, our reflections indicate a number of factors which enable such projects to be successful, namely: building participants' capacities by training as well as doing action planning; empowering leaders who provided support and were ready to support changes to happen; families and the community joining hands together; assistance and coordination by the nurses stationed at the health facilities; the determination of the family health leaders; and the ability to adjust strategies to conform according to the roles within the families and community. These findings are consistent with the theory of health changing performances at the primary level, involving changes such as role aspects, leader functions and facilities, as shown in a research report by Merzel and D'Afflitti (2003) and by Bhagabaty and others (2015).

Reflecting more widely, the key to this approach seems to lie in "community mobilization" and related processes that emphasize participation and enhance 
'social capital' and personal networks (Sherraden et al., 2002; Freudenberg et al., 2011). Clearly in the context of our studies in North East Thailand, this is underpinned by a dynamic nursing model which is being used and developed to enable such mobilisation. Findings from family health nurses themselves, highlighted recently by Jongudomkarn and Macduff (2014), show that the KKU Family Health Nursing Model can enable three main strategies for optimal care delivery, namely: enacting a participatory action approach mobilising families' social capital; using family nursing process; and implementing action strategies within communities. Informed by the WHO Europe Family Health Nursing approach, this involves family nurses stationed in towns and villages using holistic practices and knowledge in performing their roles and functions within the four dimensions of care: Health Promotion/Prevention, Early Detection, Direct/ Care, and Rehabilitation.

Moreover this holistic care covers physical, mental, social, and spiritual aspects. Our project operation processes have shown that family nursing roles are involved in every aspect, supporting many kinds of participation and from different units to achieve health care in the community. They can work independently, using social capital for operation and establishing strong family and community cooperation. Health promotion moves forward by the communities developing and maintaining these important roles. Such an aspiration was incorporated at strategic level in the Thai national health plan development, volume 11 , during the years 2012-2016, proving the importance that the role of family nursing plays in the primary health care services system. Following the assigned roles will enable the vision of the national health plan development, volume 11 , to be achieved through the years 2012-2016.

Limitations: although this research was conducted in a real community setting there were a number of distinctive perspectives, especially pertaining to Thai culture. Close relationships in the community are a particular feature of Thai culture and traditions. Such communitybased participatory campaigns for responsible alcohol consumption and the creation of consumption guidelines might be considered a violation of personal rights in Western societies where people may tend to regard alcohol consumption as their own personal business. Nevertheless, there is considerable scope for further research to evaluate and refine the approach, testing applications in various contexts in Thailand and in other Asian Pacific countries and cultures.

In conclusion, the evidence from the village voices elicited in this research supports a growing body of qualitative research in this region of Thailand that suggests the value of a community mobilisation approach to prevention, detection and treatment of detrimental health issues, underpinned by the KKU Family Health Nursing Model. In this particular paper, the value of the approach in addressing alcohol consumption and thereby contributing to prevention of cancer and of long term illness has been highlighted. The lessons learned now give opportunity to further test and develop the approach in Thailand and other Asian Pacific countries.

\section{References}

Arora D, Marya CM, Menon I, et al (2015). Cross sectional survey on association between alcohol, betel- nut, cigarette consumption and health promoting behavior of industrial workers in Ghaziabad. Asian Pac J Cancer Prev, 16, 139-44.

Bhagabaty SM, Kataki AC, Kalita M, Salkar S (2015). Community based intervention for tobacco cessation: a pilot study experience, north east India. Asian Pac J Cancer Prev, 16, 811-4.

Bryman A (2001). Social research methods. Oxford University Press, Oxford.

Center for Alcohol Studies (CAS) Board. (2010). Alcohol consumption situation. Center for Alcohol Studies Press, Nontaburi [in Thai].

Center for Alcohol Studies (CAS) Board. (2013). Alcohol consumption situation. Center for Alcohol Studies Press, Nontaburi [in Thai].

Center for Alcohol Studies (CAS) Board. (2014). Status of alcohol consumption. center for alcohol studies press, Nontaburi [in Thai].

Chindaprasirt J, Sookprasert A, Wirasorn K et al (2012). Cost of colorectal cancer care in hospitalized patients of Thailand. J Med Assoc Thai, 95, 196-200.

de Menezes RF, Bergmann A, Thuler LCS (2013). Alcohol consumption and risk of cancer: a systematic literature review. Asian Pac J Cancer Prev, 14, 4965-72.

Freudenberg N, Pastor M, Israel B (2011). Strengthening community capacity to participate in making decisions to reduce disproportionate environmental exposures. Am J Public Health, 101, 123-30.

Gupta B, Johnson NW (2014). Emerging and established global life-style risk factors for cancer of the upper aero-digestive tract. Asian Pac J Cancer Prev, 15, 5983-91.

Hetherington, S (2011). How to know: a practicalist conception of knowledge, Wiley-Blackwell, Online Library.

Jongudomkarn D, Baokumphai Y, Chumpan C et al (2013a). Results of knowledge translation into action: application of the KKU family health nursing model toward knowledge, attitudes in practices or prevention, caring, and rehabilitation of stroke. J Nurs Sci Health, 36, 9-24.

Jongudomkarn D, Phulthong S, Phongsiri K et al (2013b).The experiences of practice according to the KKU family health nursing model for reducing alcohol drinking problems in communities: a qualitative study of the family health leaders. J Nurs Sci Health, 36, 81-94.

Jongudomkarn D (2014). A volunteer alcohol consumption reduction campaign: participatory action research among Thai women in the Isaan region. Asian Pac J Cancer Prev, 15, 7343-50.

Jongudomkarn D, Macduff C (2014). Development of a family nursing model for prevention of cancer and other noncommunicable diseases through an appreciative inquiry. Asian Pac J Cancer Prev. 15, 10367-74.

Leenharattanarak P, Lertkhachonsuk R (2014). Quality of life in gestational trophoblastic neoplasia patients after treatment in Thailand. Asian Pac J Cancer Prev, 15, 10871-4.

Lewis CC, Hurd J (2011). Lesson study step by step: how teacher learning communities improve Instruction. Heinemann, Portsmouth, $\mathrm{NH}$.

Lincoln YS, Guba EG (1985). Naturalistic inquiry. Sage, Beverly Hills, CA.

Lizárraga MLSA, Baquedano MTSA, Cardelle-Elawar M (2007). Factors that affect decision making: gender and age differences. Factors that affect decision making: gender and age differences. Intern Jour Psych Psychol Ther, 7, 381-91. McMorris BJ, Catalano RF, Kim MJ et al (2011). Influence of 
family factors and supervised alcohol use on adolescent alcohol use and harms: similarities between youth in different alcohol policy contexts. J Stud Alcohol Drugs, 72, 418-42.

Merzel C, D’Afflitti J (2003). Reconsidering community-based health promotion: promise, performance, and potential. Am $J$ Public Health, 93, 557-74.

Morgan DL (1998). The focus group guide book, Sage, London. Oh C-M, Jun J-K, Suh M (2014). Risk of cancer mortality according to the metabolic health status and degree of obesity. Asian Pac J Cancer Prev, 15, 10027-31.

Schäfer G (2011). Family functioning in families with alcohol and other drug addiction, Soc Policy J NZ, 37, 1-17.

Sherraden MS, Slosar B, \& Sherraden M (2002). Innovation in social policy: collaborative policy advocacy. Soc Work, 47, 209-21.

Silapakij P, Kittirattanapaibul P (2009). AUDIT. WHO, CAS, Thailand Minister of Public Health Office, Bangkok [In Thai].

Silverman D (2000). Doing qualitative research. Sage, London.

Wirasorn K, Suwanrungruag K, Wiangnon S et al (2012). Numbers of new cases and trends of cancer 1993-2012: Srinagarind hospital based population, Khon Kaen, northeast Thailand. Asian Pac J Cancer Prev, 15, 8423-7.

World Health Organization Europe (2000). The family health nurse: context, conceptual, framework, and curriculum. WHO Regional Office for Europe, Copenhagen.

World Health Organization (WHO). 2005. Alcohol, gender, and drinking problems. WHO Press, Geneva.

World Health Organization (WHO). (2010). Global status report on noncommunication diseases 2010, World Health Organization Press, Geneva. 